PHYSICAL REVIEW D 86, 043010 (2012)

\title{
Generalizing the running vacuum energy model and comparing with the entropic-force models
}

\author{
Spyros Basilakos, ${ }^{1, *}$ David Polarski, ${ }^{2,3, \dagger}$ and Joan Solà ${ }^{4,5, \ddagger}$ \\ ${ }^{1}$ Academy of Athens, Center for Astronomy and Applied Mathematics, Athens, Greece \\ ${ }^{2}$ Université Montpellier 2, Laboratoire Charles Coulomb UMR 5221, Montpellier F-34095, France \\ ${ }^{3}$ CNRS, Laboratoire Charles Coulomb UMR 5221, Montpellier F-34095, France \\ ${ }^{4}$ High Energy Physics Group, Department ECM, Universitat de Barcelona, \\ Avinguda Diagonal 647, Barcelona E-08028, Catalonia, Spain \\ ${ }^{5}$ Institut de Ciències del Cosmos (ICC), Universitat de Barcelona, Avinguda Diagonal 647, \\ Barcelona E-08028, Catalonia, Spain
}

(Received 24 April 2012; published 15 August 2012)

\begin{abstract}
We generalize the previously proposed running vacuum energy model by including a term proportional to $\dot{H}$, in addition to the existing $H^{2}$ term. We show that the added degree of freedom is very constrained if both low redshift and high redshift data are taken into account. Best-fit models are undistinguishable from $\Lambda \mathrm{CDM}$ at the present time, but could be distinguished in the future with very accurate data at both low and high redshifts. We stress the formal analogy at the phenomenological level of the running vacuum models with recently proposed dark energy models based on the holographic or entropic point of view, where a combination of $\dot{H}$ and $H^{2}$ term is also present. However those particular entropic formulations which do not have a constant term in the Friedmann equations are not viable. The presence of this term is necessary in order to allow for a transition from a decelerated to an accelerated expansion. In contrast, the running vacuum models, both the original and the generalized one introduced here contain this constant term in a more natural way. Finally, important conceptual issues common to all these models are emphasized.
\end{abstract}

DOI: 10.1103/PhysRevD.86.043010

PACS numbers: 95.36.+x, 04.62.+v, 11.10.Hi

\section{INTRODUCTION}

The longstanding dark energy (DE) problem was originally presented in the form of the cosmological constant (CC) problem [1]. Whichever way it is formulated, the CC problem appears as a tough polyhedric conundrum which involves many faces: not only the problem of understanding the tiny current value of the DE density $\rho_{\mathrm{DE}}$ in the context of quantum field theory (QFT) or string theory, but also the cosmic coincidence problem, i.e., why the density of matter $\rho_{m}$ is now so close to $\rho_{\mathrm{DE}}$. Dynamical DE models are helpful in order to improve the situation. They can appear in different formulations of fundamental physics. Popular possibilities are, among others, quintessence and phantom energy in its various forms [2], and scalar-tensor models [3]. Furthermore, modified gravity is another very interesting option, which has been intensively explored in the recent literature; see e.g., Refs. [4-6].

But a class of cosmic accelerating models which we wish to explore in this paper is that of dynamical vacuum energy models. They have been proposed since long agosee e.g., Refs. [7-9] and references therein. Some of these "running" vacuum models are a possible clue for tackling one or more aspects of the CC problem. Despite the various

\footnotetext{
*svasil@academyofathens.gr

david.polarski@univ-montp2.fr

†sola@ecm.ub.es
}

phenomenological existing studies of time evolving vacuum models [10], some of them are expected on more fundamental grounds e.g., within the context of QFT in curved space-time $[8,9]$. In fact, it is difficult to conceive an expanding universe with a strictly constant value of the vacuum energy density $\rho_{\Lambda}=\Lambda /(8 \pi G)$, namely one that has remained unchanged since the origin of time. It is much more natural to expect that the vacuum energy is a dynamical quantity as the Universe itself, and thereby sensitive to time evolving functions such as the Hubble rate $H=H(t)$ or the scale factor $a=a(t)=(1+z)^{-1}\left(a_{0}=1\right)$. In these models, the need for scalars is obviated and nevertheless a phenomenologically viable description for the dynamical nature of the vacuum energy is achieved. Not only so, some of these models have been successfully tested against the latest cosmological data; see e.g., the recent studies $[11,12]$. Remarkably, some particular formulations of them have been used to improve both the cosmic coincidence problem [13] and the tough "old CC problem", i.e., the fine-tuning problem-see e.g., the recent attempts within the context of modified gravity [6].

More recently, Verlinde [14] proposed that the gravitational field equations can be derived from the second law of thermodynamics in a way that would render the gravity force quite literally as a kind of "entropic force" (which is certainly not the case in e.g., Jacobson's [15] and Padmanabhan's approaches [16], in which the entropic formulation is much more general). When Verlinde's 
entropic version is applied to cosmology, the DE does not exist anymore as such, but is mimicked in an effective way by the acceleration associated to the entropic force acting outwards the cosmic horizon. It is this particular formulation that can be called "entropic-force cosmology" which was first explored in Refs. [17,18] and later on by various authors-see e.g., Refs. [19-21]. We emphasize that these models seem to lead to the same effective Friedmann equations as the aforementioned running vacuum models [7-9] with the notable difference that some of these entropic-force models, but not necessarily all of its versions, do not yield a constant term in their Friedmann equations.

The plan of the paper is as follows. In Sec. II we review the running vacuum model followed by a comparative discussion with the entropic-force models, and we emphasize the analogy at the level of the equations of motion. In Sec. III we present the background cosmology for these models. We show that the entropic-force cosmology appears as a particular case of the generalized running vacuum model. After comparing and fitting them to the data in Sec. IV, we provide our discussion and final conclusions in Sec. V.

\section{RUNNING VACUUM ENERGY AND ENTROPIC-FORCE MODELS}

As mentioned in Sec. I, dynamical dark energy is an attractive possibility in order to explain certain aspects of the cosmological constant problem. In this section we review the idea of running vacuum energy, which was suggested in the literature long ago [22], and we take opportunity to compare it with the more recent notion of entropic dark energy, specially some recent formulations of it $[17,18]$. They are formally similar but present also important differences which lead to significant phenomenological implications. The latter will be analyzed in subsequent sections.

\section{A. Running vacuum energy as dynamical dark energy}

The running vacuum energy in QFT in curved spacetime derives from the renormalization group (RG) equation suggested in the literature for $\rho_{\Lambda}$ (see Ref. [7] and references therein):

$$
\begin{aligned}
\frac{d \rho_{\Lambda}(\mu)}{d \ln \mu^{2}} & =\frac{1}{(4 \pi)^{2}}\left[\sum_{i} B_{i} M_{i}^{2} \mu^{2}+\sum_{i} C_{i} \mu^{4}+\sum_{i} \frac{D_{i}}{M_{i}^{2}} \mu^{6}+\ldots\right] \\
& \equiv n_{2} \mu^{2}+\mathcal{O}\left(\mu^{4}\right),
\end{aligned}
$$

where $M_{i}$ are the masses of the particles contributing in the loops, and $B_{i}, C_{i}, \ldots$ are dimensionless parameters. The Eq. (2.1.1) gives the rate of change of the quantum effects on the CC as a function of the scale $\mu$. Only the "soft-decoupling" terms of the form $\sim M_{i}^{2} \mu^{2}$ remain in practice, as the $M_{i}^{4}$ ones would trigger a too fast running of the cosmological term. ${ }^{1}$ The approximate integrated form of (2.1.1) is very simple:

$$
\rho_{\Lambda}(H)=n_{0}+n_{2} H^{2}+\mathcal{O}\left(H^{4}\right)
$$

where, following the aforesaid works, we have set $\mu=H$ as the characteristic mass scale for Friedmann-LemaitreRobertson-Walker (FLRW)-like universes, and we will neglect the (much) smaller higher-order powers of $H$. Indeed, notice that only even powers are allowed by the general covariance, and hence no other $H^{2 n}$-terms beyond $H^{2}$ (not even $\mathrm{H}^{4}$ ) can contribute significantly on the rhs of Eq. (2.1.2) at any stage of the cosmological history below the GUT scale $M_{X} \lesssim M_{P}$, so that we omit them. The additive constant term $n_{0}$ in (2.1.2) appears in a natural way in this framework upon integrating the RG equation. It will play a fundamental role in our discussion. Both $n_{0}$ and $n_{1}$ become related by the boundary condition $\rho_{\Lambda}\left(H_{0}\right)=\rho_{\Lambda}^{0}$, which is to be satisfied by (2.1.2) at present, $H_{0}=H\left(t_{0}\right)$. As a result these coefficients can be conveniently rewritten as follows:

$$
n_{0}=\rho_{\Lambda}^{0}-\frac{3 \nu}{8 \pi} M_{P}^{2} H_{0}^{2}, \quad n_{2}=\frac{3 \nu}{8 \pi} M_{P}^{2},
$$

where from (2.1.1) we have defined the important dimensionless parameter

$$
\nu=\frac{1}{6 \pi} \sum_{i} B_{i} \frac{M_{i}^{2}}{M_{P}^{2}}
$$

This parameter provides the main coefficient of the $\beta$-function for the running of the vacuum energy. The coefficients $B_{i}$ in (2.1.4) can be computed from the quantum loop contributions of fields with masses $M_{i}$, and hence $\nu$ is naturally expected to be nonvanishing and small $(|\nu| \ll 1)$. For instance, for GUT fields with masses $M_{i}$ near $M_{X} \sim 10^{16} \mathrm{GeV}$, a natural estimate lies in the approximate range $\nu=10^{-5}-10^{-3}$ [8]. As a result we also expect a mild running of $\rho_{\Lambda}$, hence a dynamical DE framework which is healthfully close to the well tested concordance $\Lambda$ CDM model in which $\rho_{\Lambda}$ is strictly constant. This particular situation is retrieved only for $\nu=0$, for which $\rho_{\Lambda}=\rho_{\Lambda}^{0}$ at all times. However, there is no obvious reason for $\nu$ to be strictly vanishing in QFT in curved space-time. Therefore in the general case we should have a time evolution law for the vacuum energy (2.1.2), whose leading contribution can be presented as follows:

\footnotetext{
${ }^{1}$ The main contribution to the running of $\rho_{\Lambda}$ clearly comes from the heaviest fields in a typical grand unified theory (GUT) near the Planck scale, i.e., those with masses $M_{i} \sim M_{X} \lesssim M_{P}$. See e.g., Ref. [8] for a specific scenario within this class of models, where the one-loop contribution for the $B_{i}$ coefficients is explicitly given.
} 


$$
\rho_{\Lambda}(H)=\rho_{\Lambda}^{0}+\frac{3 \nu}{8 \pi} M_{P}^{2}\left(H^{2}-H_{0}^{2}\right) .
$$

Substituting (2.1.5) in the general acceleration law for a FLRW-like universe in the presence of a vacuum energy density $\rho_{\Lambda}$, we find

$$
\begin{aligned}
\frac{\ddot{a}}{a} & =-\frac{4 \pi G}{3}\left(\rho_{m}+3 p_{m}-2 \rho_{\Lambda}\right) \\
& =-\frac{4 \pi G}{3}\left(1+3 \omega_{m}\right) \rho_{m}+C_{0}+\nu H^{2},
\end{aligned}
$$

with

$C_{0}=\frac{8 \pi G}{3} \rho_{\Lambda}^{0}-\nu H_{0}^{2}=\frac{\Lambda}{3}-\nu H_{0}^{2}=H_{0}^{2}\left(\Omega_{\Lambda}^{0}-\nu\right)$.

Here $\omega_{m}=p_{m} / \rho_{m}$ is the equation of state for a generic component of matter $\left(\omega_{m}=0\right.$ and $1 / 3$ for nonrelativistic and relativistic matter, respectively), and $\Omega_{\Lambda}^{0}=\Lambda /\left(3 H_{0}^{2}\right)$ is the cosmological $\mathrm{CC}$ parameter whose observational value is $\Omega_{\Lambda}^{0} \simeq 0.73$. We note the presence of the constant term $C_{0} \propto n_{0}$. As warned before, this term will play an important role in our study.

\section{B. Entropic-force models and effective dark energy}

It is interesting that an effective dynamical dark energy component similar to the one derived in the previous section can also be motivated within the context of the entropic models. In a particular version of this framework, called the entropic-force models [14], the holographic screen is thought to induce a force $\mathbf{F}=T \nabla S$ on a test particle near the screen, where $T$ is the temperature of the screen and $\nabla S$ is the change of entropy associated with the information contained in it [which involves a large number of degrees of freedom (d.o.f.)]. The screen is supposed to increase its entropy when the test particle approaches it. Therefore, $\nabla S$ and the normal $\mathbf{n}$ on the screen (pointing towards the particle, located in the inner volume bounded by the screen) have opposite signs. Since the force is directed towards the screen we have $F=-T d S / d r$, with $d r$ the distance of the nearby particle to the screen. When applied to cosmology [17], the entropy of the Hubble horizon $R_{H}=c / H$ is obtained from Bekenstein's formula $S_{H}=A_{H} k_{B} / 4 l_{P}^{2}$, where $A_{H}=4 \pi R_{H}^{2}$ is the area of the horizon and $l_{P}^{2}=G \hbar / c^{3}$ is the Planck's length squared. ${ }^{2}$ The change of entropy when the radius of the horizon increases by $d r$ is simply $d S_{H}=2 \pi\left(R_{H} k_{B} / l_{P}^{2}\right) d r$. Inserting it in the formula for the pressure exerted by the entropic force on the cosmological expansion,

\footnotetext{
${ }^{2}$ Note that for the sake of better clarity, we keep $\hbar$ and $c$ in this section, but natural units $\hbar=1=c$ for the rest.
}

$P=F / A=-(T / A) d S_{H} / d r$, and estimating that the horizon temperature is $T=\left(\hbar / k_{B}\right)(H / 2 \pi)$ (proportional to the de Sitter temperature)[17], one finally obtains $P=-(2 / 3) \rho_{c} c^{2}$, where $\rho_{c}=3 H^{2} /(8 \pi G)$ is the critical density. The minus sign in the pressure is of course the characteristic feature of the accelerated expansion in this entropic version. Apart from some coefficients that depend on the estimations made, and which are not essential for the argument, the basic result is that $P \propto-\rho_{\mathrm{DE}}^{\mathrm{entr}}$, where $\rho_{\mathrm{DE}}^{\text {entr }} \sim H^{2} M_{P}^{2}$ (with $M_{P}=G^{-1 / 2}$ ) is the quantity that plays the role of effective $\mathrm{DE}$ in this entropic model. This framework suggests that the entropic force leads to an effective DE density which is dynamical: it specifically evolves as the square of the Hubble rate. ${ }^{3}$ By Friedmann's equation, it immediately follows that at the present time the value of $\rho_{\mathrm{DE}}^{\mathrm{entr}}$ would be predicted in the ballpark of the measured vacuum energy density: $\rho_{\mathrm{DE}}^{\mathrm{entr}}\left(t_{0}\right) \sim H_{0}^{2} M_{P}^{2} \sim$ $\rho_{\Lambda}^{0} \sim 10^{-47} \mathrm{GeV}^{4}$, where $\rho_{\Lambda}^{0}=\Lambda /(8 \pi G)$.

Since the previous (entropic inspired) result is essentially a surface effect from the horizon, one may think of fully generalizing it by considering the gravitational action for space-times with boundaries [25]. This is achieved by adding the boundary action term $I_{B}$ to the standard Einstein-Hilbert action, $I_{\mathrm{EH}}$, namely:

$$
I_{\mathrm{EH}}+I_{B}=\frac{1}{16 \pi G} \int_{\mathcal{M}} d^{4} x \sqrt{|g|} R+\frac{1}{8 \pi G} \int_{\partial \mathcal{M}} d^{3} y \sqrt{|h|} K .
$$

Here $h$ is the determinant of the metric $h_{a b}$ on the boundary $\partial \mathcal{M}$, induced by the bulk metric $g_{\mu \nu}$ of $\mathcal{M}$, and $y^{a}$ are the coordinates on $\partial \mathcal{M}$. Furthermore, $K$ is the trace of the second fundamental form (or extrinsic curvature); if $n^{\mu}$ is the normal on the boundary, it can be written as $K=\nabla_{\mu} n^{\mu}$. The complete action is $I=I_{\mathrm{EH}}+I_{B}+I_{m}$, where $I_{m}$ represents the ordinary matter contribution. As a mere technicality, let us point out that the precise definition of the boundary term $I_{B}$ should actually include an overall sign, which is plus or minus depending on whether the hypersurface $\partial \mathcal{M}$ is space-like $\left(n^{\mu} n_{\mu}=+1\right)$ or timelike $\left(n^{\mu} n_{\mu}=-1\right)$, respectively. We exclude null surfaces for this consideration. Notice that the precise coefficient in front of the boundary integral $I_{B}$ is chosen in such a way that the surface terms generated from the metric variation of $I_{\mathrm{EH}}$ are exactly canceled by the metric variation of $I_{B}$, provided the variation $\delta g^{\mu \nu}$ is performed in such a way that it vanishes on $\partial \mathcal{M}$, i.e., provided the induced metric $h_{a b}$ on the boundary is held fixed. It follows that, in the presence of $I_{B}$, the standard form of Einstein's equations is preserved even if the space-time has boundaries.

\footnotetext{
${ }^{3}$ Apart from the running vacuum model of Sec. II A, other frameworks involving the dynamical term $\sim H^{2}$ (treated as the full DE density or as a component of it) were suggested in Ref. [23] and more recently in Ref. [24], all of them involving the idea of vacuum fluctuations.
} 
The authors of Ref. [17] presumably used the above interpretation of $I_{B}$ as a way to generalize the entropic force argument given before, in the following way. As the surface terms emerging from the variation of $I_{\mathrm{EH}}$ are canceled by $\delta I_{B}$, they assumed that if the total action would not contain $I_{B}$ the contribution of the aforementioned surface terms to the field equations would be of the order of the effect induced on them by $I_{B}$, estimated as $R$ times the prefactor $1 /(8 \pi G)$ in $I_{B}$, i.e., $\left(12 H^{2}+6 \dot{H}\right) /$ $(8 \pi G)$-evaluated in the FLRW metric, in which $H=\dot{a} / a$ and $\dot{H}=d H / d t$. However, since this is probably just a rough estimate of the effect, they finally proposed to generalize the corresponding acceleration equation for the scale factor in the form:

$$
\frac{\ddot{a}}{a}=-\frac{4 \pi G}{3}\left(1+3 \omega_{m}\right) \rho_{m}+C_{H} H^{2}+C_{\dot{H}} \dot{H} .
$$

However not all of the models considered in Ref. [17] are of this type. ${ }^{4}$ The new ingredients are $C_{H}$ and $C_{\dot{H}}$, which are certain (presumably small) dimensionless coefficients to be fitted to the observational data. Let us also mention that there can be higher-order quantum corrections on the rhs of Eq. (2.2.2)—cf. [18]. We have neglected these effects for the present discussion because they have no impact for virtually any time in the history of the Universe after inflation. This is in line with our approximation of ignoring the $\mathcal{O}\left(H^{4}\right)$ quantum corrections also in the running vacuum model discussed before.

Let us point out that the field Eq. (2.2.2) is not necessary derived from a fundamental action. Let us recall that in the most general entropic-holographic formulations, gravity is conceived as an emergent phenomenon [16], and in this sense the gravitational field equations need not necessarily be deducible from a fundamental action at the present macroscopic level of description, even though the field equations themselves may provide a fully satisfactory account of all the basic phenomena known to date. From this point of view, the ultimate origin of gravity may lie in some fundamental degrees of freedom quite different from the metric variables, namely degrees of freedom which are completely unknown to us at present [16]. If so, the field equations under discussion in this paper could just be effective field equations falling in this category and therefore no fundamental action to derive them would be needed. A detailed discussion on this point goes beyond the scope of the present work.

The quantities $\dot{H}$ and $H^{2}$ appearing on the rhs of (2.2.2) are related through $\dot{H}=-(q+1) H^{2}$ where $q$ is the deceleration parameter. During some stages of the cosmic evolution when $q$ is roughly constant, $\dot{H}$ and $H^{2}$ are approximately proportional. For example, $q \simeq 1$ for the radiation dominated epoch, and $q \simeq 1 / 2$ for the matter dominated epoch. Hence $\dot{H} \simeq-2 H^{2}$ deep in the radiation

${ }^{4}$ G. Smoot (private communication). dominated era and $\dot{H} \simeq-(3 / 2) H^{2}$ deep in the matter dominated epoch. When we compare the entropic Eq. (2.2.2) with the corresponding Eq. (2.1.6) in the running vacuum model, we see that deep in the matter dominated epoch we can set the correspondence $\nu \leftrightarrow$ $C_{H}-3 C_{\dot{H}} / 2$ between the two models. However this is not valid at low redshifts when the Universe goes over from matter domination to accelerated expansion. In this interval where type Ia supernovae (SNIa) data are located, $q$ experiences a sharp model-dependent variation. Therefore the addition of the term $C_{\dot{H}} \dot{H}$ is a genuine extension of the original running vacuum energy model.

In view of the close analogy between these models, in the next section we consider a generalization of the running vacuum model with the inclusion of a term $\dot{H}$ together with the $H^{2}$ one.

\section{BACKGROUND SOLUTION OF THE COSMOLOGICAL FIELD EQUATIONS}

In this section we consider the solution of the cosmological field equations for both the generalized running vacuum model and the entropic-force model. We discuss in detail the underlying local conservation laws of matter and radiation in interaction with a dynamical vacuum energy component and we show that this leads to important conceptual issues. Finally, we emphasize the crucial importance of a constant term which rules out some of the entropic-force models lacking this term.

\section{A. The generic cosmological framework}

The cosmological equations of both the running vacuum models and the entropic-force models can be solved in a common framework. We will consider spatially flat FRLW cosmologies

$$
d s^{2}=d t^{2}-a^{2}(t) d \mathbf{x}^{2} .
$$

Hence the (expansion) dynamics are fully encoded in the time evolution of the scale factor $a(t)$. Instead of obeying the usual Friedmann equations of general relativity, our models obey modified Friedmann equations, viz.

$$
\begin{array}{r}
\left(\frac{\dot{a}}{a}\right)^{2}=\frac{8 \pi G}{3} \sum_{i} \rho_{i}+C_{0}+C_{H} H^{2}+C_{\dot{H}} \dot{H}, \\
\frac{\ddot{a}}{a}=-\frac{4 \pi G}{3} \sum_{i}\left(\rho_{i}+3 p_{i}\right)+C_{0}+C_{H} H^{2}+C_{\dot{H}} \dot{H},
\end{array}
$$

where the remaining sum is over the matter components only. For realistic cosmologies, we take as usual two components, namely nonrelativistic (dustlike) matter with $p_{m}=w_{m} \rho_{m}=0$ and radiation with $p_{r}=w_{r} \rho_{r}=\frac{1}{3} \rho_{r}$. These equations can be viewed formally as resulting from the presence of a time-dependent component $\rho_{\Lambda}(t)=$ $\rho_{\Lambda}(H(t), \dot{H}(t))$ satisfying 


$$
\rho_{\Lambda}(H, \dot{H})=\frac{3}{8 \pi G}\left(C_{0}+C_{H} H^{2}+C_{\dot{H}} \dot{H}\right)=-p_{\Lambda}(H, \dot{H}) .
$$

We will call this dynamical component a "generalized running vacuum energy" (GRVE) density since its equation of state satisfies $w_{\Lambda}=p_{\Lambda} / \rho_{\Lambda}=-1$ as in the case of a strictly constant vacuum energy. ${ }^{5}$ By the same token we will call the class of these models with $C_{0} \neq 0$ the generalized running vacuum models. In the particular case $C_{\dot{H}}=0$ we recover the original running vacuum model discussed in Sec. II A. Formally, the generalization of the model being proposed here implies that the scale $\mu^{2}$ in Eq. (2.1.1), which is to be eventually associated with a physical quantity according to the RG procedure, should in general be a linear combination of $H^{2}$ and $\dot{H}$ rather than just the $H^{2}$ component, as these two terms represent independent d.o.f. with the same dimension. Finally, let us emphasize that the particular case $C_{0}=0$ is not to be included within the class of GRVE models because the integration of the RG Eq. (2.1.1) always involves an additive term leading to $C_{0} \neq 0$. The case $C_{0}=0$ seems to appear in some of the entropic-force models [17] briefly addressed in the previous subsection. While this setting can be derived as a particular case of our general analysis of the system (3.1.2) and (3.1.3), we stress that $C_{0}=0$ leads to a qualitatively new situation, which we will comment in subsequent sections and that is not expected from the conceptual point of view of the running vacuum model framework. With these provisos in mind we are now going to solve the background cosmology of the entire class of models (3.1.2) and (3.1.3).

\section{B. Discussion of the local conservation laws}

Once the metric (3.1.1) is given, a comoving perfect fluid with energy-momentum tensor $T_{\mu \nu}=$ $\left(\rho_{i}+p_{i}\right) u_{\mu} u_{\nu}-p_{i} g_{\mu \nu}$ will satisfy the conservation equation $\dot{\rho}_{i}=-3 H\left(\rho_{i}+p_{i}\right)$, with $\rho_{i}$ and $p_{i}$ appearing in Eqs. (3.1.2) and (3.1.3) provided $\rho_{\Lambda}$ is constant. This applies of course for nonflat FLRW universes as well. However, if the vacuum energy density is a time-dependent component it cannot have this energy-momentum tensor. Actually, as we will see now the same applies for the other components appearing in Eqs. (3.1.2) and (3.1.3).

However, from the system (3.1.2) and (3.1.3) we get the coupled conservation equation

$$
\dot{\rho}_{m}+\dot{\rho}_{r}+\dot{\rho}_{\Lambda}=-3 H \rho_{m}-4 H \rho_{r} .
$$

\footnotetext{
${ }^{5}$ Let us notice that the recent work [26], which extends the discussion of a model first suggested in Appendix C of Ref. [11], contains a linear term in $H$ rather than our $\dot{H}$ term. As mentioned in Sec. II A, odd powers of $H$ cannot emerge from a covariant effective action, and in this sense these models are more phenomenological than the class of GRVE models (3.1.4) presented here.
}

This equation is indeed a first integral of that system. Clearly, for $\dot{\rho}_{\Lambda} \neq 0$ none of the components can satisfy the standard conservation equation as emphasized above. ${ }^{6}$

It is easy to see that this does not depend on the particular choice (3.1.4). Let us assume that the variable vacuum has an energy density $\frac{8 \pi G}{3} \rho_{\Lambda}(t)=C_{H} H^{2}$ and that it is a perfect fluid with an a priori undefined equation of state. Then it follows from its (assumed) conservation equation that its equation of state parameter $w_{\Lambda}$ satisfies $-\frac{\dot{H}}{H^{2}}=\frac{3}{2} \times$ $\left(1+w_{\Lambda}\right)$. So if we consider a simple (flat) universe containing also dust, the only consistent way is to have $\Omega_{\Lambda}=C_{H}=1$. So we end with no dust at all and the first Friedmann equation reduces to an equality (while $w_{\Lambda}$ remains of course undefined). We stress that these conceptual issues are also true for other models with similar effective Friedmann equations, for example, models inspired by the holographic principle or models based on the entropic-force principle. Ultimately these properties arise from the absence (in general) of a formulation at the level of the action which is still an open issue.

As mentioned in the previous section, in a pragmatic approach we assume the validity of (3.1.2) and (3.1.3) without explicitly deducing them from an underlying action-see, however, [8] for a specific framework along these lines. After some calculations the coupled conservation equation (3.2.1) reads

$$
\begin{gathered}
\dot{\rho}_{m}+\dot{\rho}_{r}-\frac{3}{2} C_{\dot{H}}\left(\dot{\rho}_{m}+\frac{4}{3} \dot{\rho}_{r}\right) \\
=-3 H\left(1-C_{H}\right)\left(\rho_{m}+\frac{4}{3} \rho_{r}\right) .
\end{gathered}
$$

We note that the model does not yield a conservation equation for each component separately, seemingly overlooked in Ref. [17]. For this we would need to specify the action of the model and to find the corresponding energymomentum tensors. Moreover, the system is not fully defined by Eqs. (3.1.2) and (3.1.3). Indeed, any solution of the following set of equations

$$
\begin{gathered}
\dot{\rho}_{m}=-3 H \frac{1-C_{H}}{1-\frac{3}{2} C_{\dot{H}}} \rho_{m}+Q, \\
\dot{\rho}_{r}=-4 H \frac{1-C_{H}}{1-2 C_{\dot{H}}} \rho_{r}-Q,
\end{gathered}
$$

\footnotetext{
${ }^{6}$ The nonconservation of matter in the presence of running vacuum energy has recently been proposed as a possible link between the dynamical $\mathrm{DE}$ and the increasing evidence for a possible variation of the fundamental constants and scales in nature, as e.g., the QCD scale-see Ref. [27]. However, a running vacuum energy of the form (2.1.5) can be made compatible with matter conservation if one allows $G$ to slowly evolve with time, see Ref. [8] for a concrete scenario connected with an action functional. For the present GRVE framework, though, $G$ is assumed to be strictly constant.
} 
for arbitrary function $Q=Q(t)$, will be a solution of Eq. (3.2.2). In the matter dominated $\left(\rho_{r} \approx 0\right)$ and radiation dominated $\left(\rho_{m} \approx 0\right)$ stages we must have from Eq. (3.2.2) that $Q \rightarrow 0$. The simplest version of this model is to assume that Eq. (3.2.2) reduces at all times to a set of decoupled equations with $Q=0$ at all times. We conjecture that this is perhaps the only way to introduce consistently an arbitrary number of species.

It will be convenient to introduce the following notations:

$$
\begin{gathered}
\nu \equiv C_{H}, \\
\alpha \equiv \frac{3}{2} C_{\dot{H}},
\end{gathered}
$$

as well as the important quantities

$$
\begin{gathered}
\xi_{m} \equiv \frac{1-\nu}{1-\alpha}, \\
\xi_{r} \equiv \frac{1-\nu}{1-\frac{4}{3} \alpha} .
\end{gathered}
$$

The motivation for the relabeling (3.2.5) is simply because for $C_{\dot{H}}=0$ the GRVE model boils down to the original running vacuum model discussed in Sec. II A, and then $C_{H}$ exactly reduces to the parameter $\nu$ defined in that section. Using these definitions, Eqs. (3.2.3) and (3.2.4) (setting $Q=0$ ) can be written as follows:

$$
\begin{gathered}
\dot{\rho}_{m}=-3 H \xi_{m} \rho_{m}, \\
\dot{\rho}_{r}=-4 H \xi_{r} \rho_{r},
\end{gathered}
$$

for which it is straightforward to obtain the corresponding solutions (setting $a_{0}=1$ ):

$$
\begin{gathered}
\rho_{m}=\rho_{m}^{0} a^{-3 \xi_{m}}=\rho_{m}^{0}(1+z)^{3 \xi_{m}}, \\
\rho_{r}=\rho_{r}^{0} a^{-4 \xi_{r}}=\rho_{r}^{0}(1+z)^{4 \xi_{r}} .
\end{gathered}
$$

Note that these decoupled solutions reduce automatically to the behavior of dustlike matter during matter domination $\left(\rho_{r} \approx 0\right)$ and to the radiation component during radiation domination $\left(\rho_{m} \approx 0\right)$. They take the standard form for $\xi_{m}=1$ and $\xi_{r}=1$.

\section{Determining the time evolving vacuum energy and the Hubble function}

The Eqs. (3.2.9) and (3.2.10) are decoupled; there is no transfer of energy between the two components. However there is a transfer of energy between the running vacuum energy $\rho_{\Lambda}$ and these components. We find for the evolution of $\rho_{\Lambda}$

$$
\dot{\rho}_{\Lambda}=3 H\left(\xi_{m}-1\right) \rho_{m}+4 H\left(\xi_{r}-1\right) \rho_{r} .
$$

When $\xi_{m}=\xi_{r}=1$ the standard behavior of matter and radiation is recovered and then $\rho_{\Lambda}=\Lambda /(8 \pi G)$ reduces to a genuine cosmological constant with $\Lambda=3 C_{0}$. The transfer of energy between the matter components and the GRVE is the physical reason for the particular scaling behaviors (3.2.11) and (3.2.12), which obviously depart from the standard expectations $\rho_{m} \sim a^{-3}$ and $\rho_{r} \sim a^{-4}$ in the $\Lambda \mathrm{CDM}$ owing to the nonvanishing values of the parameters $\nu$ and $\alpha$. We have here an effective interacting dark energy model. Consistency enforces to have an interaction between the running vacuum energy and all other components.

The addition of the term $C_{\dot{H}} \dot{H}=\frac{2}{3} \alpha \dot{H}$ introduces an important change compared to the original running vacuum model discussed in Sec. II Ain which $C_{\dot{H}}=0$ but the new degree of freedom is severely constrained by observations. The reason is that the model cannot depart too much from the $\Lambda \mathrm{CDM}$ values $\xi_{m}=\xi_{r}=1$, with

$$
\xi_{r}=\xi_{m} \frac{1-\alpha}{1-\frac{4}{3} \alpha} .
$$

The only way to satisfy both constraints $\xi_{m} \approx 1$ and $\xi_{r} \approx 1$ is to have

$$
|\nu| \ll 1, \quad|\alpha| \ll 1 \Leftrightarrow \xi_{m} \approx 1, \quad \xi_{r} \approx 1 .
$$

Of course we have $\xi_{m}=\xi_{r}=1$ when both parameters $\nu$ and $\alpha$ vanish. Note also that the condition $\xi_{r} \approx 1$ is crucial for the viability of our model at high redshifts [e.g., when fitting the model against cosmic microwave background (CMB) data]. If we use only constraints at very low redshifts, models with $\xi_{m} \approx 1$ (i.e., $|\nu| \ll 1$ ) but not necessarily satisfying $|\alpha| \ll 1$ will fare well in this domain, though the model would actually be unviable taking into account its behavior at high redshifts.

Compared to the old running vacuum energy model $(\alpha=0)$, the generalized model offers more possibilities to depart from standard cosmology:

(i) One can have $\xi_{r}=\xi_{m} \approx 1$, in which case the model just reduces to the original running model ( $\alpha=0$ or $C_{\dot{H}}=0$, and $\nu \neq 0$ ). Both radiation and dust scale in a nonstandard way but their departure from standard behavior is not independent and depends on one single parameter $\nu$, specifically $\rho_{m} \sim a^{-3(1-\nu)}$ and $\rho_{r} \sim a^{-4(1-\nu)}$.

(ii) $\xi_{r}=1$ and $\xi_{m} \approx 1$, in which case radiation behaves in the standard way but dust does not. This occurs when $\nu=\frac{4}{3} \alpha \neq 0$. This case exists only in the generalized model $\alpha \neq 0$. Departure from standard cosmology occurs already at low redshifts.

(iii) $\xi_{m}=1$ and $\xi_{r} \approx 1$, in which dust scales in standard way but radiation does not. This corresponds to $\nu=\alpha \neq 0$. This case can mimic standard cosmology at low redshifts but it is strongly constrained when high redshift data are considered. 
(iv) $\xi_{m} \approx 1, \xi_{r} \approx 1$ and $\xi_{m} \neq \xi_{r}$, in which the deviation of the nonrelativistic component is different from the relativistic one, and hence this provides an extension of the first case discussed above which is only possible within the GRVE model.

The three last cases above are only possible in the generalized model $\alpha \neq 0$. However these additional possibilities are strongly constrained by observations on both low and high redshifts. We will see in particular in Sec. IV that the strong constraint on $\alpha$ in the regime (3.3.3) is similar to that one found for $\nu$ in the original running model — see the recent analyses [11,12].

Equation (3.3.1) is easily recast in the form

$$
\frac{d \rho_{\Lambda}}{d a}=3\left(\xi_{m}-1\right) \frac{\rho_{m}}{a}+4\left(\xi_{r}-1\right) \frac{\rho_{r}}{a}
$$

which is easily integrated using the solutions (3.2.11) and (3.2.12) and the explicit form of the vacuum energy as a function of the scale factor can be expressed as follows:

$$
\begin{aligned}
\rho_{\Lambda}(a)= & \rho_{\Lambda}^{0}+\rho_{m}^{0}\left(\xi_{m}^{-1}-1\right)\left(a^{-3 \xi_{m}}-1\right) \\
& +\rho_{r}^{0}\left(\xi_{r}^{-1}-1\right)\left(a^{-4 \xi_{r}}-1\right) .
\end{aligned}
$$

The Hubble function can now be constructed from the matter components (3.2.9) and (3.2.10) and the vacuum energy (3.3.5):

$$
\begin{aligned}
H^{2} & =\frac{8 \pi G}{3}\left[\rho_{m}+\rho_{r}+\rho_{\Lambda}\right] \\
& =H_{0}^{2}\left[\Omega_{m}^{0} \frac{\rho_{m}}{\rho_{m}^{0}}+\Omega_{r}^{0} \frac{\rho_{r}}{\rho_{r}^{0}}+\Omega_{\Lambda}^{0} \frac{\rho_{\Lambda}}{\rho_{\Lambda}^{0}}\right] .
\end{aligned}
$$

Introducing the normalized Hubble rate in terms of the redshift, $E(z) \equiv H(z) / H_{0}$, we find

$$
E^{2}(z)=\frac{\Omega_{m}^{0}}{\xi_{m}}(1+z)^{3 \xi_{m}}+\frac{\Omega_{r}^{0}}{\xi_{r}}(1+z)^{4 \xi_{r}}+\frac{H_{0}^{-2} C_{0}}{1-\nu},
$$

where we have used the standard definition $\Omega_{i}=\rho_{i} / \rho_{c}$, with $\rho_{c}=3 H^{2} /(8 \pi G)$, satisfying the constraint

$$
\Omega_{\Lambda}+\Omega_{m}+\Omega_{r}=1
$$

at all times.

Note that the boundary condition $E(z=0)=1$ in Eq. (3.3.7) leads to the equality

$$
H_{0}^{-2} C_{0}=\Omega_{\Lambda}^{0}-\Delta \nu,
$$

where we have defined $\Delta \nu=\nu-\bar{\nu}$, with $\bar{\nu}=$ $\alpha \Omega_{m}^{0}+(4 / 3) \alpha \Omega_{r}^{0}$. The $\bar{\nu}$ parameter is characteristic of the extension of the original running vacuum model into the GRVE model and is closely related to $\nu$. Indeed $\bar{\nu}$ gauges the size of the new $\dot{H}$-effect in terms of $H^{2}$ at the present time since it satisfies the relation $C_{\dot{H}} \dot{H}_{0}=-\bar{\nu} H_{0}^{2}$, which can be compared to $C_{H} H_{0}^{2}=\nu H_{0}^{2}$ in the original running model. To confirm that relation let us write the current value of the $C_{\dot{H}} \dot{H}$ term in the starting Eqs. (3.1.2) and (3.1.3) as follows:

$$
C_{\dot{H}} \dot{H}_{0}=-C_{\dot{H}}\left(q_{0}+1\right) H_{0}^{2}=-\left(\frac{3}{2} \Omega_{m}^{0}+2 \Omega_{r}^{0}\right) C_{\dot{H}} H_{0}^{2} .
$$

Thus we find $\bar{\nu}=\bar{\nu}_{m}+\bar{\nu}_{r}$, where $\bar{\nu}_{m}=(3 / 2) \Omega_{m}^{0} C_{\dot{H}}=$ $\alpha \Omega_{m}^{0} \quad$ and $\quad \bar{\nu}_{r}=2 \Omega_{r}^{0} C_{\dot{H}}=(4 / 3) \alpha \Omega_{r}^{0} \quad$ represent the nonrelativistic and relativistic matter contributions, respectively.

After having determined the explicit relation between $\bar{\nu}$ and the other parameters, we see from Eq. (3.3.9) that $C_{0}$ becomes also explicitly determined as follows:

$$
\begin{aligned}
C_{0} & =H_{0}^{2}\left[\Omega_{\Lambda}^{0}-\nu+\left(\Omega_{m}^{0}+\frac{4}{3} \Omega_{r}^{0}\right) \alpha\right] \\
& =H_{0}^{2}\left[\Omega_{\Lambda}^{0}-C_{H}+\frac{3}{2}\left(\Omega_{m}^{0}+\frac{4}{3} \Omega_{r}^{0}\right) C_{\dot{H}}\right] .
\end{aligned}
$$

Notice that for $\alpha=0$ (or $C_{\dot{H}}=0$ ) it boils down to the corresponding expression (2.1.7) for the original running vacuum model. On the other hand Eq. (3.3.11) tells us another interesting feature, to wit: models with $C_{0}=0$ cannot have the two parameters $\nu$ and $\alpha$ (equivalently $C_{H}$ and $C_{\dot{H}}$ ) simultaneously small, i.e., it is impossible to satisfy the relations (3.3.3) unless $\Omega_{\Lambda}^{0}=0$-which is of course unacceptable. In particular, entropic-force models [17] cannot have $C_{H}$ and $C_{\dot{H}}$ simultaneously small, otherwise they would contradict the measured value of the cosmological term: $\Omega_{\Lambda}^{0} \simeq 0.73$. Even if we would accept that at least one of the parameters $C_{H}$ and $C_{\dot{H}}$ is not small, the resulting model would be contrived as it would entail a nontrivial modification of the standard $\Lambda \mathrm{CDM}$ cosmology. Actually in the next section we will encounter a related difficulty, which is perhaps the biggest stumbling block to the $C_{0}=0$ models.

\section{Crucial distinction between some entropic-force and GRVE models}

In the previous subsections we have solved in detailed the full class of cosmological models based on the set of generalized FLRW Eqs. (3.1.2) and (3.1.3). In particular, we have assumed arbitrary values for the parameters $C_{0}$, $C_{H} \equiv \nu$ and $C_{\dot{H}} \propto \alpha$. However we expect from observational constraints that the last two ones are sufficiently small-cf. Eq. (3.3.3) in order for the generalized models not to depart too much from the standard scaling laws of matter and radiation.

We turn now our attention specifically to the additive parameter $C_{0}$. If the other two parameters $\left(C_{H}\right.$ and $\left.C_{\dot{H}}\right)$ have to be small, this is not the case for $C_{0}$ and as we will see now it cannot vanish. While the running vacuum energy models have a nonvanishing $C_{0}$, this is not the case for some entropic force models. 
Indeed, successful models must be able to produce an accelerated expansion at very low redshifts. To start with let us analyze the situation $C_{0}=0$. It is easy to derive from the expression for $\frac{\ddot{a}}{a}$ that accelerated expansion is obtained both in the matter and radiation-dominated stages if the following condition is satisfied (with $C_{0}=0$ ):

$$
\nu-\frac{2}{3} \alpha>\frac{1}{2} \Leftrightarrow 2 \xi_{r}<1 .
$$

In the matter-dominated era a slightly weaker condition is required:

$$
\nu-\frac{2}{3} \alpha>\frac{1}{3} \Leftrightarrow \frac{3}{2} \xi_{m}<1
$$

We see that these conditions are redshift independent. Therefore, if we have an accelerated expansion rate at very low redshifts, we will have it at least during all of the matter-dominated stage. This leads obviously to an unviable cosmology putting aside the fact that the corresponding scaling behaviors are completely unviable observationally.

We can recover these results solving for the time dependence of $H(t)$ and $a(t)$. The following equation holds during matter domination:

$$
\dot{H}+\frac{3}{2} \xi_{m} H^{2}=\frac{3}{2} \xi_{m} \frac{C_{0}}{1-\nu} .
$$

When $C_{0}=0$ the condition (3.4.2) for accelerated expansion is clearly recovered from Eq. (3.4.3). In the general case $C_{0} \neq 0$, Eq. (3.4.3) can be solved to yield

$$
\begin{gathered}
H(t)=A \operatorname{coth}\left[\frac{3}{2} \xi_{m} A t\right], \\
a(t)=D \sinh ^{2 / 3 \xi_{m}}\left[\frac{3}{2} \xi_{m} A t\right],
\end{gathered}
$$

where we have used (3.3.9) and we have set

$$
D=\left[\frac{\left.\Omega_{m, 0}-\bar{\nu}_{M}\right)}{\Omega_{\Lambda, 0}-\Delta \nu}\right]^{1 / 3 \xi_{m}}, \quad A=H_{0} \sqrt{\frac{\Omega_{\Lambda, 0}-\Delta \nu}{1-\nu}} .
$$

Returning to the case with vanishing $C_{0}$, the solution to (3.4.3) reads

$$
a(t) \propto t^{2 / 3 \xi_{m}}, \quad \frac{\ddot{a}}{a} \sim\left(\frac{2}{3 \xi_{m}}-1\right) t^{-2},
$$

which shows again that (3.4.2) leads to accelerated expansion. Hence we conclude that models with $C_{0}=0$ cannot describe an expanding universe undergoing a transition from decelerated to accelerated expansion.

\section{E. Observational interpretation}

For an "Einsteinian" interpretation of the generalized running vacuum models, i.e., those represented by Eqs. (3.3.6) and (3.3.7) with $C_{0} \neq 0$, let us write

$$
\begin{aligned}
H^{2} & =\frac{8 \pi G}{3}\left[\tilde{\rho}_{m}+\tilde{\rho}_{r}\right]+\frac{\tilde{\Lambda}}{3} \\
& =H_{0}^{2}\left[\tilde{\Omega}_{m}^{0} \frac{\tilde{\rho}_{m}}{\tilde{\rho}_{m}^{0}}+\tilde{\Omega}_{r}^{0} \frac{\tilde{\rho}_{r}}{\tilde{\rho}_{r}^{0}}\right]+\frac{\tilde{\Lambda}}{3},
\end{aligned}
$$

with the obvious identifications

$$
\begin{gathered}
\tilde{\rho}_{m}=\frac{\rho_{m}}{\xi_{m}}, \quad \tilde{\rho}_{r}=\frac{\rho_{r}}{\xi_{r}}, \quad \frac{\tilde{\Lambda}}{3}=\frac{C_{0}}{1-\nu}, \\
\tilde{\Omega}_{m}^{0}=\frac{\Omega_{m}^{0}}{\xi_{m}}=\frac{\tilde{\rho}_{m}^{0}}{\rho_{c}^{0}}, \quad \tilde{\Omega}_{r}^{0}=\frac{\Omega_{m}^{0}}{\xi_{r}}=\frac{\tilde{\rho}_{r}^{0}}{\rho_{c}^{0}} .
\end{gathered}
$$

Observationally there is no reason to distinguish between the matter or radiation energy density appearing in the starting Eq. (3.1.2) and that part contained in $\rho_{\Lambda}$. Hence it is natural to identify the observed value $\Omega_{m \text {,obs }}^{0}$ with $\tilde{\Omega}_{m}^{0}$ and similarly $\Omega_{r \text {;obs }}^{0}$ with $\tilde{\Omega}_{r}^{0}$. We still have the standard equality valid at all times,

$$
\tilde{\Omega}_{m}+\tilde{\Omega}_{r}+\tilde{\Omega}_{\Lambda}=1,
$$

with $\tilde{\Omega}_{\Lambda}=\frac{\tilde{\Lambda}}{3 H^{2}}$.

Even recast in the form (3.5.1) we should remember that the energy densities $\tilde{\rho}_{m}$ and $\tilde{\rho}_{r}$, obey the nonstandard scaling laws (3.2.11), respectively, (3.2.12). Interestingly, in this Einsteinian interpretation, our model reduces to a model with a genuine cosmological constant $\tilde{\Lambda}$ and nonstandard evolution of dust and radiation. In the generalized running vacuum energy model, the departure from standard behavior of dust and radiation are independent from each other.

In this model, the redshift at equality $z_{\mathrm{eq}}$ is given by

$$
\left(1+z_{\mathrm{eq}}\right)^{4 \xi_{r}-3 \xi_{m}}=\frac{\tilde{\Omega}_{m}^{0}}{\tilde{\Omega}_{r}^{0}} .
$$

The variation of $z_{\mathrm{eq}}$ constrains the quantity $4 \xi_{r}-3 \xi_{m}$, a constraint that will be satisfied by our best-fit models found in next section. In view of (3.3.7) we expect further very tight constraints on $\xi_{r}$ itself deep in the radiation dominated era.

In a first conservative approach we would like to keep a standard thermal history. Even if $\tilde{\Omega}_{r}^{0}$ assumes the value for $\Omega_{r}^{0}$ required by standard big bang nucleosynthesis, and assuming that cosmic temperature scales in the standard way, the expansion rate at the big bang nucleosynthesis epoch will get changed by a nonstandard amount due to the scaling law (3.2.12). Inserting numbers this finally yields the conservative constraint $\left|\xi_{r}-1\right|<10^{-3}$ because the expansion rate is severely constrained and cannot vary too much at the time of big bang nucleosynthesis (see e.g., Ref. [28]).

As $\xi_{r}$ is a free parameter, in practice we wish to explore scenarios satisfying

$$
\xi_{r}=1
$$


This choice means that the model parameters $\nu$ and $\alpha$ are no longer independent, and from (3.2.8) we see that we must have $\alpha=3 \nu / 4$. This ensures that the standard thermal history is recovered. Indeed, with the choice (3.5.5), the radiation dominated stage in our models is essentially similar to the standard radiation dominated stage. We have in particular that the temperature of thermalized relativistic species scales consistently in the standard way. This is in particular true for the CMB temperature.

We have derived all equations necessary in order to constrain with observations the class of generalized FLRW models (3.1.2) and (3.1.3) satisfying Eq. (3.5.5). This we do in the next section.

\section{FITTING THE MODELS TO THE OBSERVATIONAL DATA}

In the following we present some details of the statistical method and on the observational samples and data statistical analysis that will be adopted to constrain the models presented in the previous sections. We shall extract our fit from the combined data on SNIa, the data on the baryonic acoustic oscillations (BAOs), and the shift parameter of the CMB. Note that in the case of the BAO analysis we have to modify it appropriately in order to incorporate some specific features of the present models.

\section{A. The global fit to SNIa, BAOs and CMB}

First of all, we use the Union 2 set of 557 type Ia supernovae of Amanullah et al. [29]. ${ }^{7}$ The corresponding $\chi^{2}$-function to be minimized is

$$
\chi_{\mathrm{SNIa}}^{2}(\mathbf{p})=\sum_{i=1}^{557}\left[\frac{\mu_{\mathrm{th}}\left(z_{i}, \mathbf{p}\right)-\mu_{\mathrm{obs}}\left(z_{i}\right)}{\sigma_{i}}\right]^{2},
$$

where $z_{i}$ is the observed redshift for each data point. The fitted quantity $\mu$ is the distance modulus, defined as $\mu \equiv m-M=5 \log d_{L}+25$, in which $d_{L}(z, \mathbf{p})$ is the luminosity distance:

$$
d_{L}(z, \mathbf{p})=(1+z) \int_{0}^{z} \frac{\mathrm{d} z^{\prime}}{H\left(z^{\prime}\right)} .
$$

Here $\mathbf{p}$ a vector containing the cosmological parameters of our model that we wish to fit for. In our case one possibility would be to take e.g., $\mathbf{p}=\left(\tilde{\Omega}_{m}^{0}, \nu\right)$. In Eq. (4.1.1), the theoretically calculated distance modulus $\mu_{\text {th }}$ for each point follows from Eq. (4.1.2), in which the Hubble function $H(z)=H_{0} E(z)$ is given by Eq. (3.3.7) for the generic model under consideration. Finally, $\mu_{\mathrm{obs}}\left(z_{i}\right)$ and $\sigma_{i}$ stand for the measured distance modulus and the corresponding $1 \sigma$ uncertainty for each SNIa data point, respectively. The previous Eq. (4.1.2) for the luminosity distance applies only for spatially flat universes, which we are assuming

\footnotetext{
${ }^{7}$ Note that the data can be found in at http://supernova.lbl.gov/ Union/.
}

throughout. Note that since only the relative distances of the SNIa are accurate and not their absolute local calibration, we always marginalize with respect to the internally derived Hubble constant (for methods that do not need to a priori marginalize over the internally estimated Hubble constant, see for example Refs. [30,31]). In the case of the Union2 SNIa data the internally derived Hubble constant is $H_{0} \simeq 70 \mathrm{Km} / \mathrm{s} / \mathrm{Mpc}$ which is in agreement to that of WMAP7 [32] $H_{0}=70.4 \mathrm{Km} / \mathrm{s} / \mathrm{Mpc}$ used in the present study.

In addition to the SNIa data, we also consider the BAO scale produced in the last scattering surface by the competition between the pressure of the coupled baryonphoton fluid and gravity. The resulting acoustic waves leave (in the course of the evolution) an overdensity signature at certain length scales of the matter distribution. Evidence of this excess has been found in the clustering properties of the SDSS galaxies (see Refs. [33-35]) and it provides a "standard ruler" that we can employ to constrain dark energy models. In this work we use the results of Percival et al. [34], $r_{s}\left(z_{d}\right) / D_{\mathrm{V}}\left(z_{\star}\right)=0.1390 \pm 0.0037$. Note that $r_{s}\left(z_{d}\right)$ is the comoving sound horizon size at the baryon drag epoch [36] (i.e., the epoch at which baryons are released from the Compton drag of photons), and $z_{d} \sim \mathcal{O}\left(10^{3}\right)$ is the corresponding redshift of that epoch, closely related to that of last scattering-the precise expression being given by the fitting formula of Ref. [36]. Finally, $D_{V}(z)$ is the effective distance measure [33] and $z_{\star}=0.275$. Of course, the quantities $\left(r_{s}, D_{\mathrm{V}}\right)$ can be defined analytically. In particular, $r_{s}\left(z_{d}\right)$ is given by the comoving distance that light can travel prior to redshift $z_{d}$ :

$$
r_{s}\left(z_{d}\right)=\int_{0}^{t\left(z_{d}\right)} \frac{c_{s} d t}{a}=\int_{0}^{a_{d}} \frac{c_{s}(a) d a}{a^{2} H(a)}
$$

where $a_{d}=\left(1+z_{d}\right)^{-1}$, and

$$
c_{s}(a)=\left(\frac{\delta \tilde{p}_{\gamma}}{\delta \tilde{\rho}_{\gamma}+\delta \tilde{\rho}_{b}}\right)^{1 / 2}=\frac{1}{\sqrt{3(1+\mathcal{R}(a))}}
$$

is the sound speed in the baryon-photon plasma. Here we assume adiabatic perturbations and we have used $\delta \tilde{p}_{b}=0$ and $\delta \tilde{p}_{\gamma}=(1 / 3) \delta \tilde{\rho}_{\gamma}$, and defined $\mathcal{R}(a)=\delta \tilde{\rho}_{b} / \delta \tilde{\rho}_{\gamma}$. If the scaling laws for nonrelativistic matter and radiation would be those of the standard model, we would have $\mathcal{R}(a)=3 \rho_{b} / 4 \rho_{\gamma}$, which can be finally cast as a linear function of the scale factor $\mathcal{R}(a)=\left(3 \Omega_{b}^{0} / 4 \Omega_{\gamma}^{0}\right) a$, where $\Omega_{b}^{0} h^{2} \simeq 0.02263$ and $\Omega_{\gamma}^{0} h^{2} \simeq 2.47 \times 10^{-5}$ are the current values of the normalized baryon and photon densities. However, our scaling laws for nonrelativistic matter and radiation are given by Eqs. (3.2.11) and (3.2.12). As a result, the sound speed velocity in the plasma gets a correction with respect to the standard result $\left(a_{0}=1\right)$ : 


$$
\mathcal{R}(a)=\frac{3}{4} \frac{\xi_{m}}{\xi_{r}} \frac{\tilde{\rho}_{b}(a)}{\tilde{\rho}_{\gamma}(a)}=\frac{3}{4} \frac{1-4 \alpha / 3}{1-\alpha} \frac{\tilde{\Omega}_{b}^{0}}{\tilde{\Omega}_{\gamma}^{0}} a^{4 \xi_{r}-3 \xi_{m}} .
$$

Of course for $\nu=0$ and $\alpha=0\left(\xi_{m}=\xi_{r}=1\right)$ the previous equation becomes again a linear function of the scale factor, and it exactly reduces to the standard result.

The remaining ingredients of the $\mathrm{BAO}$ analysis are as in the standard case, in particular the effective distance is (see Ref. [33])

$$
D_{\mathrm{V}}(z) \equiv\left[(1+z)^{2} D_{A}^{2}(z) \frac{c z}{H(z)}\right]^{1 / 3},
$$

where $D_{A}(z)=(1+z)^{-2} d_{L}(z, \mathbf{p})$ is the angular diameter distance. Therefore, the corresponding $\chi_{\mathrm{BAO}}^{2}$ function is simply written as

$$
\chi_{\mathrm{BAO}}^{2}(\mathbf{p})=\frac{\left[\frac{r_{s}\left(z_{d}\right)}{D_{\mathrm{V}}\left(z_{\star}\right)}(\mathbf{p})-0.1390\right]^{2}}{0.0037^{2}} .
$$

Furthermore, a very accurate and deep geometrical probe of dark energy is the angular scale of the sound horizon at the last scattering surface, as encoded in the location $l_{1}^{T T}$ of the first peak of the CMB temperature perturbation spectrum. This probe is described by the CMB shift parameter [37,38], defined as

$$
R=\sqrt{\Omega_{m}^{0}} \int_{0}^{z_{l s}} \frac{d z}{E(z)}
$$

The measured shift parameter according to the WMAP 7-year data [32] is $R=1.726 \pm 0.018$ at the redshift of the last scattering surface: $z_{l s}=1091.36$. In this case, the $\chi^{2}$-function is given by

$$
\chi_{\mathrm{CMB}}^{2}(\mathbf{p})=\frac{[R(\mathbf{p})-1.726]^{2}}{0.018^{2}} .
$$

For a detailed discussion of the shift parameter as a cosmological probe, see e.g., Ref. [39]. Let us emphasize that when dealing with the CMB shift parameter we have to include both the matter and radiation terms in the total normalized matter density entering the $E(z)$ function in Eq. (4.1.8), given explicitly by Eq. (3.3.7). Indeed, the radiation contribution reads $\Omega_{r}^{0}=\left(1+0.227 N_{\nu}\right) \Omega_{\gamma}^{0}$, with $N_{\nu}$ the number of neutrino species. Therefore, at $z_{l s}=1091.36$, and including three light neutrino species, the radiation contribution amounts to $\sim 24 \%$ of the total energy density associated to matter, which is not negligible. We use $h=0.704$ in our analysis.

Our statistical analysis, due to its simplicity, has been used extensively in the literature in order to constrain the dark energy models (see for example Refs. [31,40,41] and references therein). We would like to point that a more general statistical presentation would require the covariances of BAO and CMB shift parameter. We have checked our statistical results using the latter covariances and our results remain the same as they should. Note that the corresponding covariances can be found in Percival et al. [34] and in Komatsu et al. [32], respectively. Finally, as emphasized before Eq. (3.1.1) we restrict our analysis to spatially flat spaces. This seems justified in view of the tight constraints on $\Omega_{k, 0}$ and is sufficient for our purposes.

\section{B. Numerical results}

Since we perform an overall fit of the SNIa + BAO + CMB data, it is important to take into account the contribution of both nonrelativistic matter and radiation.

(i) For the concordance $\Lambda \mathrm{CDM}$ cosmology, we simply have $\rho_{\Lambda}^{0}=$ const. and

$$
\rho_{m}(z)=\rho_{m}^{0}(1+z)^{3}, \quad \rho_{r}(z)=\rho_{r}^{0}(1+z)^{4} .
$$

(ii) Concerning the generalized running vacuum energy model (3.1.2) and (3.1.2) we have explicitly given the corresponding density formulas in Sec. III. Let us recall that $C_{0}=0$ is a very particular case that we exclude from the class of the GRVE models. We have shown in the previous section that this case is not viable observationally and therefore we will not consider it any further for the phenomenological analysis. Therefore, from now on we assume that $C_{0} \neq 0$ and focus on fitting the parameters of this model to the SNIa $+\mathrm{BAO}+\mathrm{CMB}$ data. In particular, we already know that $\nu$ and $\alpha$ have to be small—see Eq. (3.3.3) — but only the direct confrontation of the model with the data will tell us about their possible maximum size. In practice, considering models satisfying (3.5.5), it will be convenient to define the effective parameter

$$
\nu_{\text {eff }} \equiv \nu-\alpha=\frac{1}{4} \nu,
$$

and use $\nu_{\text {eff }}$ as fitting parameter, together with $\tilde{\Omega}_{m}^{0}$. We can check this explicitly by expanding $\xi_{m}$ and $\xi_{r}$ linearly in $|\nu| \ll 1$ and $|\alpha| \ll 1$, together with some coefficients in Eq. (3.3.5), and using the definition (4.2.2):

$$
\xi_{m} \simeq 1-\nu_{\text {eff }}, \quad \xi_{r}=1 .
$$

As a result, for the energy densities we find

$$
\begin{aligned}
\tilde{\rho}_{m}(z) & =\tilde{\rho}_{m}^{0}(z)(1+z)^{3\left(1-\nu_{\text {eff }}\right)}, \\
\tilde{\rho}_{r}(z) & =\tilde{\rho}_{r}^{0}(1+z)^{4} .
\end{aligned}
$$

Similarly the corresponding normalized Hubble flow squared reads

$$
E^{2}(z)=\tilde{\Omega}_{m}^{0}(1+z)^{3\left(1-\nu_{\text {eff }}\right)}+\tilde{\Omega}_{r}^{0}(1+z)^{4}+\tilde{\Omega}_{\Lambda}^{0} .
$$

Finally, let us mention that within the same approximation we can write the BAO ratio (4.1.5) entering 
the modified sound speed of the baryon-photon plasma as follows:

$$
\mathcal{R}(a)=\frac{3}{4}\left(1-\nu_{\mathrm{eff}}\right) \frac{\tilde{\Omega}_{b}^{0}}{\tilde{\Omega}_{\gamma}^{0}} a^{1+3 \nu_{\text {eff }}} .
$$

For $\nu=0$ and $\alpha=0$ (hence $\nu_{\text {eff }}=0$ ) it clearly reduces to the standard result mentioned in the previous subsection.

These formulas confirm our contention that we can fully reexpress all the background formulas in terms of the effective fitting vector

$$
\mathbf{p}_{\text {eff }}=\left(\tilde{\Omega}_{m}^{0}, \nu_{\text {eff }}\right) .
$$

We also see from the previous formulas that $\nu_{\text {eff }}$ is the single effective parameter that controls the deviations of the GRVE model with respect to the $\Lambda$ CDM model in the low $z$ region (when radiation can be neglected). It is only in the high redshift region where the model is sensitive to independent contributions from $\nu_{\text {eff }}$ (equivalently, from $\nu$ or $\alpha$ ). Notice that this feature could be used, in principle, to distinguish between the two sorts of running models, i.e., the original one (which we reviewed briefly in Sec. II A) and the generalized running vacuum model under discussion in this paper. At low $z$ the two kinds of models are indistinguishable because they both depend on a single parameter, $\nu$ and $\nu_{\text {eff }}$, respectively.

Let us next proceed with the numerical fit analysis. In order to place tighter constraints on the corresponding parameter space of our model, the probes described above must be combined through a joint likelihood analysis $^{8}$ given by the product of the individual likelihoods according to

$$
\mathcal{L}_{\text {tot }}(\mathbf{p})=\mathcal{L}_{\text {SNIa }} \times \mathcal{L}_{\mathrm{BAO}} \times \mathcal{L}_{\mathrm{CMB}} .
$$

Since likelihoods are defined as $\mathcal{L}_{j} \propto \exp \left(-\chi_{j}^{2} / 2\right)$, it translates into an addition for the joint $\chi^{2}$ function:

$$
\chi_{\mathrm{tot}}^{2}(\mathbf{p})=\chi_{\mathrm{SNIa}}^{2}+\chi_{\mathrm{BAO}}^{2}+\chi_{\mathrm{CMB}}^{2} .
$$

In our $\chi^{2}$ minimization procedure, for the vacuum models (running and concordance $\Lambda \mathrm{CDM}$ ) we use the following range and steps for the fitting parameters: $\tilde{\Omega}_{m}^{0} \in[0.01,1]$ in steps of 0.001 and $\nu_{\text {eff }} \in[-0.02,0.02]$ in steps of $10^{-4}$. The numerical results that we obtain are the following. In the case of the generalized running vacuum model the overall likelihood function peaks at $\tilde{\Omega}_{m}^{0}=0.274 \pm 0.011$, $\nu_{\text {eff }}=-0.00133 \pm 0.0028 \quad\left(\right.$ or $\quad \nu=4 \nu_{\text {eff }} \simeq-0.00532$, $\left.\alpha=3 \nu_{\text {eff }} \simeq-0.004\right) \quad$ with $\quad \chi_{\text {tot }}^{2}\left(\tilde{\Omega}_{m}^{0}, \nu_{\text {eff }}\right) \simeq 542.93$ for

\footnotetext{
${ }^{8}$ Likelihoods are normalized to their maximum values. In the present analysis we always report $1 \sigma$ uncertainties on the fitted parameters. Note also that the total number of data points used here is $N_{\text {tot }}=559$, while the associated degrees of freedom is: d.o. $f=N_{\text {tot }}-n_{\text {fit }}-1$, where $n_{\text {fit }}$ is the model-dependent number of fitted parameters.
}

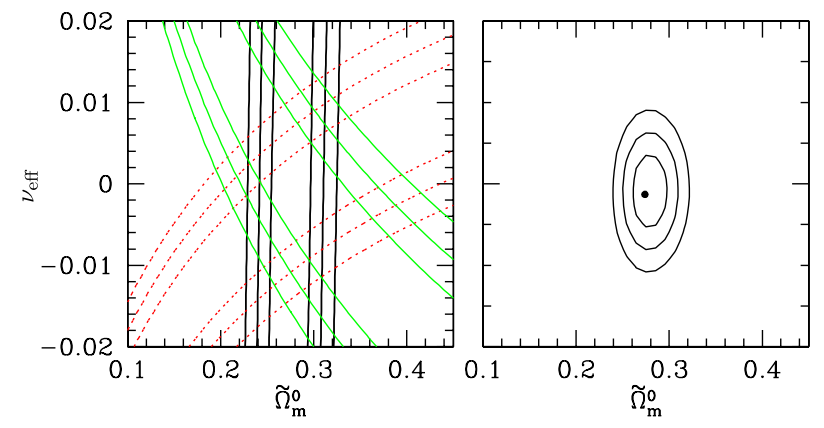

FIG. 1 (color online). Likelihood contours (for $-2 \ln \mathcal{L} / \mathcal{L}_{\max }$ equal to $2.30,6.16$ and 11.81, corresponding to $1 \sigma, 2 \sigma$ and $3 \sigma$ confidence levels) in the $\left(\tilde{\Omega}_{m}^{0}, \nu_{\text {eff }}\right)$ plane for the generalized running vacuum model (4.2.5) $\left(C_{0} \neq 0\right.$ or $\left.\tilde{\Omega}_{\Lambda}^{0} \neq 0\right)$. For the $\mathrm{CMB}$ analysis we include also the radiation component as indicated in Eqs. (4.2.4) and (4.2.5). The left panel shows the contour lines based on the SNIa data: thick solid, almost vertical lines (in black); BAOs: dotted curvilinear lines (in red); and CMB shift parameter: solid curvilinear lines (in green). In the right panel we show the corresponding contours based on the joint statistical analysis of the SNIa + BAO + CMB data.

556 degrees of freedom. ${ }^{9}$ In Fig. 1 we present the $1 \sigma, 2 \sigma$ and $3 \sigma$ confidence levels in the $\left(\tilde{\Omega}_{m}^{0}, \nu_{\text {eff }}\right)$ plane. In particular, the left panel in that figure shows the individual likelihood contours, with the SNIa-based results indicated by thick solid lines, the BAO results by dotted red lines and those based on the CMB shift parameter by solid curvilinear lines (in green). Using the SNIa data alone it is evident that although the $\tilde{\Omega}_{m}^{0}$ parameter is tightly constrained $(\simeq 0.27)$, the $\nu_{\text {eff }}$ parameter remains completely unconstrained. As can be seen in the right plots of Fig. 1, the above degeneracy is broken when using the joint likelihood analysis, involving all the cosmological data. Finally, in the case of the concordance $\Lambda \mathrm{CDM}$ cosmology $\left(\nu_{\text {eff }}=\alpha \equiv 0\right)$ we find $\Omega_{m}^{0}=0.274 \pm 0.01$ with $\chi_{\text {tot }}^{2}\left(\Omega_{m}^{0}\right) /$ d.o.f $\simeq 543.18 / 558$.

Overall we see that the departure of the GRVE model with respect to the $\Lambda \mathrm{CDM}$ is extremely small and cannot be detected at present.

\section{DISCUSSION AND CONCLUSIONS}

In this paper we have generalized the running vacuum energy models and we have solved the corresponding background cosmology. The generalized running vacuum model (3.1.2), (3.1.3), and (3.1.4) with $C_{0} \neq 0$ is able to pass the SNIa + BAO + CMB data constraints with a statistical significance comparable to that of the concordance $\Lambda$ CDM model which is a limiting case of the model $\left(\xi_{r}=\xi_{m}=1\right.$, or equivalently $\alpha=\nu=0$ ). Although the best-fit models are currently indistinguishable from

\footnotetext{
${ }^{9}$ Note that in Ref. [12] the original running vacuum model was used-see Eqs. (2.1.5) and (2.1.6) - in which $\alpha$ is strictly equal to zero- and the Constitution set of 397 SNIa data [42]. We would like to mention here that those results for $\nu$ are in agreement with the current results for $\nu_{\text {eff }}$ within $1 \sigma$ uncertainties.
} 
$\Lambda \mathrm{CDM}$ we expect that future very accurate data on both low and high redshifts could help to distinguish these models from the standard cosmology.

Some conceptual issues pertaining to these models were also addressed which are related to the peculiar conservation laws derived in Sec. III. We stress that these issues hold as well for other models with analogous effective Friedmann equations, models inspired either by the holographic or the entropic-force principle. We have further emphasized that the presence of a nonvanishing additive constant $C_{0}$ is crucial since otherwise the cosmology does not allow for a transition between decelerated and accelerated expansion. That was actually noticed in previous entropic-force studies [20]. ${ }^{10}$ In contrast, the class of the running vacuum models, both the generalized one (GRVE) presented here and the original one (which existed in the literature since long ago-see Ref. [7] and references therein) do not suffer from this problem because $C_{0}$ is naturally expected to be nonvanishing as a result of integrating the corresponding RG equation. Therefore, despite the formal analogies between these two sorts of models, the running vacuum models are naturally well positioned for a correct phenomenological description of our cosmos.

From the point of view of the running vacuum models, the current Universe appears as FLRW-like with a genuine cosmological constant while dust and radiation evolve in a nonstandard way, in the sense that they follow scaling laws that deviate slightly from their behavior in $\Lambda$ CDM. In contrast to the old running vacuum energy model, the generalized one introduced in this paper allows for an independent departure from the standard behavior of both components. We have used this freedom and we have explored models satisfying $\xi_{r}=1$ thereby ensuring that relativistic matter obeys the standard behavior. In this way

\footnotetext{
${ }^{10}$ For other problems related with the entropic-force cosmology, see the recent Ref. [43].
}

potential difficulties related to the radiation dominated era are essentially avoided. While the other parameter $\xi_{m} \simeq 1-\nu_{\text {eff }}$ remains free, it can be efficiently constrained using CMB data. It is constrained by observations at a similar level as the single parameter $\nu$ of the original running model, i.e., they are both presently allowed up to $\mathcal{O}\left(10^{-3}\right)$ at most (in absolute value).

This order of magnitude size is consistent with the theoretical expectations on these coefficients, interpreted as one-loop $\beta$-functions of the running cosmological constant. The mild variation induced on the CC term by these coefficients is responsible for the dynamical character of the vacuum energy, which is of course the reason why these models have a chance to improve the situation with the $\Lambda \mathrm{CDM}$ without giving up its phenomenological success. Such time variation is foreseen on general QFT grounds and it provides a possible formulation of an effective dynamical dark energy, which in some cases can help curing the cosmic coincidence problem [44] and other related problems.

To summarize, the running vacuum models offer a challenging phenomenologically consistent description of a universe with presently accelerated expansion. The dynamical $\Lambda$ could be understood in the context of QFT in curved space-time. Such potential connection with fundamental physics could help to conceive the origin of a dynamical $\Lambda$ term in QFT and eventually provide an explanation for the tough cosmological constant problem.

\section{ACKNOWLEDGMENTS}

J.S. has been supported in part by MEC and FEDER under Grant No. PA2010-20807, by the Spanish program CPAN CSD2007-00042 and by 2009SGR502 Generalitat de Catalunya. S. B. thanks the Department of ECM of the University de Barcelona for the hospitality, and the financial support from the Spanish Ministerio de Education, within the Project No. SAB2010-0118.
[1] S. Weinberg, Rev. Mod. Phys. 61, 1 (1989); V. Sahni and A. Starobinsky, Int. J. Mod. Phys. D 9, 373 (2000); T. Padmanabhan, Phys. Rep. 380, 235 (2003).

[2] For a review, see e.g., P. J.E. Peebles and B. Ratra, Rev. Mod. Phys. 75, 559 (2003); E. J. Copeland, M. Sami, and S. Tsujikawa, Int. J. Mod. Phys. D 15, 1753 (2006); V. Sahni and A. Starobinsky, Int. J. Mod. Phys. D 15, 2105 (2006); M. Li, X.-D. Li, S. Wang, and Y. Wang, Commun. Theor. Phys. 56, 525 (2011); D. Weinberg, M. Mortonson, D. Eisenstein, C. Hirata, A. Riess, and E. Rozo, arXiv:1201.2434.

[3] B. Boisseau, G. Esposito-Farèse, D. Polarski, and A. A. Starobinsky, Phys. Rev. Lett. 85, 2236 (2000).
[4] S. Nojiri and S. D. Odintsov, eConf C0602061, 06 (2006); Int. J. Geom. Methods Mod. Phys. 4, 115 (2007); T. P. Sotiriou and V. Faraoni, Rev. Mod. Phys. 82, 451 (2010); R. Woodard, Lect. Notes Phys. 720, 403 (2007); S. Capozziello and M. De Laurentis, Phys. Rep. 509, 167 (2011).

[5] L. Amendola, R. Gannouji, D. Polarski, and S. Tsujikawa, Phys. Rev. D 75, 083504 (2007); W. Hu and I. Sawicki, Phys. Rev. D 76, 064004 (2007); A. A. Starobinsky, JETP Lett. 86, 157 (2007); S. A. Appleby and R. A. Battye, Phys. Lett. B 654, 7 (2007).

[6] F. Bauer, J. Solà, and H. Štefančić, J. Cosmol. Astropart. Phys. 12 (2010) 029; Phys. Lett. B 688, 269 (2010); 
Mod. Phys. Lett. A 26, 2559 (2011); Phys. Lett. B 678, 427 (2009); F. Bauer, Classical Quantum Gravity 27, 055001 (2010); 28, 225019 (2011); J. Solà, Fortschr. Phys. 59, 1108 (2011); S. Basilakos, F. Bauer, and J. Solà, J. Cosmol. Astropart. Phys. 01 (2012) 050.

[7] J. Solà, J. Phys. Conf. Ser. 283, 012033 (2011).

[8] J. Solà, J. Phys. A 41, 164066 (2008).

[9] I. L. Shapiro and J. Solà, Phys. Lett. B 682, 105 (2009); confer also to the extended version arXiv:0808.0315, and references therein.

[10] O. Bertolami, Nuovo Cimento B 93, 36 (1986); M M. Ozer and O. Taha, Nucl. Phys. B287, 776 (1987); O. K. Freese, F. C. Adams, J. A. Frieman, and E. Mottola, Nucl. Phys. 287, 797 (1987); J. C. Carvalho, J. A. S. Lima, and I. Waga, Phys. Rev. D 46, 2404 (1992); see e.g., the reviews J. M. Overduin and F. I. Cooperstock, Phys. Rev. D 58, 043506 (1998); R. G. Vishwakarma, Classical Quantum Gravity 18, 1159 (2001), and references therein.

[11] S. Basilakos, M. Plionis, and J. Solà, Phys. Rev. D 80, 083511 (2009).

[12] J. Grande, J. Solà, S. Basilakos, and M. Plionis, J. Cosmol. Astropart. Phys. 08 (2011) 007.

[13] J. Grande, J. Solà, and H. Štefančić, J. Cosmol. Astropart. Phys.08 (2006) 011; Phys. Lett. B 645, 235 (2007); J. Phys. A 40, 6787 (2007).

[14] E. P. Verlinde, J. High Energy Phys.04 (2011) 029.

[15] T. Jacobson, Phys. Rev. Lett. 75, 1260 (1995).

[16] For a review, see e.g., T. Padmanabhan, Rep. Prog. Phys. 73, 046901 (2010); Phys. Rep. 406, 49 (2005), and references therein.

[17] D. A. Easson, P. H. Frampton, and G. F. Smoot, Phys. Lett. B 696, 273 (2011).

[18] D. A. Easson, P. H. Frampton, and G. F. Smoot, Int. J. Mod. Phys. A 27, 1250066 (2012).

[19] R. Casadio and A. Gruppuso, Phys. Rev. D 84, 023503 (2011).

[20] T.S. Koivisto, D.F. Mota, and M. Zumalacarregui, J. Cosmol. Astropart. Phys. 02 (2011) 027.

[21] Y.-F. Cai, J. Liu, and H. Li, Phys. Lett. B 690, 213 (2010); H. Wei, Phys. Lett. B 692, 167 (2010); Y. S. Myung, Astrophys. Space Sci. 335, 553 (2011); Y.F. Cai and E. N. Saridakis, Phys. Lett. B 697, 280 (2011); M. Visser, J. High Energy Phys. 10 (2011) 140.

[22] I. L. Shapiro and J. Solà, J. High Energy Phys. 02 (2002) 006; Phys. Lett. B 475, 236 (2000); Nucl. Phys. B, Proc. Suppl. 127, 71 (2004); Proc. Sci., AHEP2003 (2003) 013; J. Solà and H. Sttefančić, Phys. Lett. B 624, 147 (2005); Mod. Phys. Lett. A 21, 479 (2006).
[23] T. Padmanabhan, Classical Quantum Gravity 22, L107 (2005).

[24] M. Maggiore, Phys. Rev. D 83, 063514 (2011); N. Bilic, Phys. Rev. D 83, 105003 (2011); L. Hollenstein, M. Jaccard, M. Maggiore, and E. Mitsou, Phys. Rev. D 85, 124031 (2012); Phys. Lett. B 704, 102 (2011); N. Bilic, S. Domazet, and B. Guberina, Phys. Lett. B 707, 221 (2012).

[25] S. W. Hawking and G. T. Horowitz, Classical Quantum Gravity 13, 1487 (1996).

[26] F.E.M. Costa, J.A.S. Lima, and F. A. Oliveira, arXiv:1204.1864.

[27] H. Fritzsch and J. Solà, arXiv:1202.5097.

[28] K. Jedamzik and M. Pospelov, New J. Phys. 11, 105028 (2009).

[29] R. Amanullah et al., Astrophys. J. 716, 712 (2010).

[30] S. Nesseris and L. Perivolaropoulos, Phys. Rev. D 72, 123519 (2005).

[31] H. Wei and R.-G. Cai, Phys. Lett. B 663, 1 (2008).

[32] E. Komatsu et al. (WMAP Collaboration), Astrophys. J. Suppl. Ser. 180, 330 (2009); Astrophys. J. Suppl. Ser. 192, 18 (2011).

[33] D. J. Eisenstein et al. (SDSS Collaboration), Astrophys. J. 633, 560 (2005); N. Padmanabhan et al. (SDSS Collaboration), Mon. Not. R. Astron. Soc. 378, 852 (2007).

[34] W. J. Percival et al. (SDSS Collaboration), Mon. Not. R. Astron. Soc. 401, 2148 (2010); E. A. Kazin, Astrophys. J. 710, 1444 (2010).

[35] C. Blake et al., Mon. Not. R. Astron. Soc. 418, 1707 (2011).

[36] D. J. Eisenstein and W. Hu, Astrophys. J. 496, 605 (1998).

[37] J. R. Bond, G. Efstathiou, and M. Tegmark, Mon. Not. R. Astron. Soc. 291, L33 (1997).

[38] S. Nesseris and L. Perivolaropoulos, J. Cosmol. Astropart. Phys. 01 (2007) 018.

[39] O. Elgaroy and T. Multamäki, Astron. Astrophys. 471, 65 (2007).

[40] T. M. Davis et al., Astrophys. J. 666, 716 (2007).

[41] M. Plionis, R. Terlevich, S. Basilakos, F. Bresolin, E. Terlevich, J. Melnick, and R. Chavez, Mon. Not. R. Astron. Soc. 416, 2981 (2011).

[42] M. Hicken, W. M. Wood-Vasey, S. Blondin, P. Challis, S. Jha, P. L. Kelly, A. Rest, and R. P. Kirshner, Astrophys. J. 700, 1097 (2009).

[43] J. J. Roveto and G. Munoz, arXiv:1201.2475.

[44] J. Grande, A. Pelinson, and J. Solà, Phys. Rev. D 79, 043006 (2009). 